\title{
Comportamento Prossocial em Adolescentes Estudantes: Uso de um Programa de Intervenção Breve
}

\author{
Rosana Cretendio Pajares \\ Maria Aznar-Farias \\ Laboratório de Psicologia Ambiental e Desenvolvimento Humano da Universidade \\ Federal de São Paulo, Santos, SP, Brasil \\ Adriana Marcassa Tucci \\ Departamento de Saúde, Educação e Sociedade, \\ Programa de Pós-Graduação Interdisciplinar em Ciências da Saúde \\ Universidade Federal de São Paulo, Santos, SP, Brasil \\ Nancy Ramacciotti de Oliveira-Monteiro ${ }^{1}$ \\ Departamento de Ciências do Mar da Universidade Federal de São Paulo, Santos, SP, Brasil \\ Coordenação do Laboratório de Psicologia Ambiental e Desenvolvimento Humano \\ da Universidade Federal de São Paulo, Santos, SP, Brasil
}

\begin{abstract}
Resumo
A adolescência é uma etapa da vida com grande potencial de incremento de habilidades e competências, dentre elas, os comportamentos prossociais. Essa possibilidade, reafirmada pela Psicologia Positiva, apresenta-se como alternativa ao pólo negativo muitas vezes expresso nos comportamentos antissociais de adolescentes. Os comportamentos prossociais abordados nesta investigação estão distribuídos nas categorias de ajuda, partilha, empatia, cuidado e atenção, e clima positivo. Eles são definidos como comportamentos que favorecem outras pessoas ou grupos sem busca de recompensa externa ou material e podem gerar reciprocidade positiva nas relações interpessoais e sociais, configurando-se como estratégias para mobilização de recursos protetivos para a saúde mental. Este artigo apresenta um estudo exploratório e descritivo que avaliou comportamentos prossociais em 21 adolescentes (13 a 15 anos), estudantes de uma escola pública do município de Santos (SP), antes e após aplicação de um programa de intervenção breve em prossocialidade. O instrumento utilizado foi a Escala de Avaliação de Prossocialidade para Adolescentes (EAP-A), além da aplicação do Programa Mínimo de Incremento Prossocial (PMIP). Os resultados foram analisados de forma quantitativa e qualitativa. A análise quantitativa indicou tendência a graus médios para comportamentos prossociais no grupo investigado, antes e após a intervenção; a análise qualitativa mostrou indicadores de melhora nos comportamentos prossociais. Ficam sugeridos novos estudos para ampliação e aprofundamento acerca da temática proposta em amostras maiores com adolescentes que apresentem queixas de problemas de comportamento e graus baixos de prossocialidade.
\end{abstract}

Palavras-chaves: Comportamentos prossociais, adolescência, desenvolvimento humano, Psicologia Positiva.

Endereço para correspondência: Universidade Federal de São Paulo, Laboratório de Psicologia Ambiental e Desenvolvimento Humano, Rua Silva Jardim, 136, $3^{\circ}$ andar, sala 327, Vila Mathias, Santos, SP, Brasil 11015020. E-mail: rosana.unifesp@gmail.com, aznar.farias@gmail.com, adritucci@uol.com.br e nancy.unifesp@ gmail.com

Agradecimento ao estatístico Fabio Montessano pelo apoio na análise quantitativa dos resultados. 


\title{
Prosocial Behavior among Adolescents: Use of a Brief Intervention Program
}

\begin{abstract}
Adolescence is a stage of life with great potential for increasing skills and competencies, among them, the prossocial behaviors. This possibility, as reaffirmed by Positive Psychology, presents itself as an alternative to the negative pole often expressed by antisocial behavior in adolescents. The prossocial behaviors addressed in this research are distributed in categories helping, sharing, empathy, care and attention, and positive mood. They are defined as behaviors that favor other individuals or groups without seeking external reward or material and can generate positive reciprocity in interpersonal and social relationships, configured as protective strategies for mobilizing resources for mental health. This article presents an exploratory and descriptive study that evaluated prossocial behaviors in 21 adolescents (1315 years), students in a public school in the city of Santos (SP), before and after application of a brief intervention program in prossociability. The instrument used was Prossociability Assessment Scale for Adolescents (EAP-A), and the application of the Minimum Prosocial Improvement Program (PMIP). The results were analyzed by qualitative and quantitative method. Quantitative analysis indicated a trend for the average degree in prossocial behaviors investigated, before and after the intervention; qualitative analysis showed indicators of improvement in prossocial behaviors. Further studies are suggested to broadening and deepening the proposed theme in larger samples with adolescents with behavior problems and lower grades of prossociability.
\end{abstract}

Keywords: Prossocial behaviors, adolescence, human development, positive psychology.

\section{Conducta Prosocial en Adolescentes Estudiantes: Uso de un Programa de Intervención Breve}

\section{Resumen}

Adolescencia es una etapa de la vida con gran potencial para el aumento de capacidades y competencias, como las conductas prosociales. Esa posibilidad, reafirmada por la Psicología Positiva, es una alternativa al polo negativo a menudo expresada por conducta antisocial en adolescentes. Los comportamientos prosociales abordados en esta investigación están distribuidos en: ayudar, compartir, empatía, cuidado y atención, y el estado de ánimo positivo. Se definen como comportamientos que favorecen a otros individuos o grupos sin buscar recompensa externa o material podiendo generar reciprocidad positiva en las relaciones interpersonales y sociales. Configuran como estrategias de protección para la movilización de recursos para la salud mental. Este artículo presenta um estúdio exploratorio y descriptivo que evaluó comportamientos prosociales en 21 adolescentes (13-15 años), en alumnos de una escuela pública en la ciudad de Santos (SP), antes y después de la aplicación de un programa de intervención breve en prosocialidad. El instrumento utilizado fué Prosocialidad Escala de Evaluación de la Adolescencia (EAP-A), y la aplicación del Programa de Mejoramiento Prosocial Mínimo (PMIP). Se analizaron los resultados de manera cualitativa y cuantitativa. El análisis cuantitativo indica una tendencia para el grado médio en comportamientos prosociales investigados, antes y después de la intervención; el análisis cualitativo mostró indicadores de mejora en comportamentos prosociales. Se sugere ampliar y profundizar sobre el tema propuesto en muestras más grandes con adolescentes con quejas de problemas de comportamiento y con calificaciones más bajas de prosocialidad.

Palabras clave: Comportamientos prossociais, adolescencia, desarrollo humano, Psicología Positiva. 
A adolescência, relevante fase de transformação, constituição e estruturação da identidade, inclui importantes transições psicossociais. Muitos adolescentes, em especial os que vivem em centros urbanos, estão inseridos em territórios permeados por violência e comportamentos antissociais. Espaços destinados ao desenvolvimento, à educação e à promoção de saúde, como as instituições de ensino, por vezes não escapam desse cenário permeado por violência. No entanto, as escolas carregam em si o valioso potencial para despertar e desenvolver valores e ações sociais positivas nos adolescentes, como os comportamentos prossociais. Segundo o Ministério da Saúde (MS, 2010), consoante com a Organização Mundial de Saúde (OMS), adolescente é a pessoa com idade entre 10 e 20 anos.

A adolescência tem sido descrita como um dos marcos mais importantes no processo de desenvolvimento humano, inserindo-se como transição entre a infância e a idade adulta, quando são estabelecidos hábitos de conduta e modelos de socialização. É nessa fase que se criam princípios da moralidade adulta, dos códigos éticos e do compromisso ideológico (Zacarés \& Serra, 1998).

A formação da identidade pessoal é característica predominante nos adolescentes e implica em autoconhecimento e conhecimento da sua história familiar em um processo reflexivo sobre valores. Essa formação se dá nas interações pessoais e na vivência de conflitos inerentes a esse período de desenvolvimento (Milani, Jesus, $\&$ Bastos, 2006), também caracterizado pela predominância das relações com o grupo de iguais e pela vivência de espaços de experimentação de novos comportamentos, pensamentos e sentimentos (Trianes, Muñoz, \& Jiménez, 1997). Do ponto de vista psicanalítico, o adolescente é um sujeito em processo de transformação, em profunda revisão de seu mundo interno e de suas "heranças" infantis, com vistas à adaptação ao novo corpo e às novas pulsões, decorrentes da puberdade. Para além das alterações corporais, a adolescência assume peculiaridades conforme a cultura vigente de um determinado momento histórico (Levy, 2001).
Em seu importante trabalho sobre as etapas do desenvolvimento psicossocial, Erikson (1990) apresenta a adolescência como uma fase do desenvolvimento humano com vivência de conflitos de ego voltados à crise de identidade $\mathrm{e}$ confusão de papéis. Essa "crise" é entendida pelo autor como oportunidade de tomada de decisões e possibilidade de construção e de escolhas de valores para a vida, na busca de realização e reconhecimento na cultura na qual o adolescente está inserido.

As alterações físicas e psíquicas vividas nesse período provocam repercussões não só no próprio adolescente, mas também nos diversos contextos ambientais em que está inserido, como a família, a escola, a comunidade e a sociedade como um todo. Problemas psicológicos podem aparecer em decorrência de falhas no controle e na interação desses diversos contextos de vida dos adolescentes, a partir de fatores de vulnerabilidade individuais, familiares e socioculturais (Bronfenbrenner, 2001). No entanto, apesar das alterações e dos possíveis problemas, a adolescência não precisa ser, necessariamente, um período problemático (Shoen-Ferreira, Aznar-Farias, \& Silvares, 2010).

Para o MS (2010), o grupo populacional formado por adolescentes e jovens no Brasil apresenta maior vulnerabilidade diante de diferentes formas de violência, por outro lado, o próprio Ministério refere também que a sustentabilidade das estratégias de saúde e de desenvolvimento comunitário depende, dentre outros fatores, da formação da capacidade de liderança, de participação e espírito de serviço à coletividade de adolescentes e jovens. Nesse sentido, experiências que priorizam a participação dos adolescentes como protagonistas do seu processo de desenvolvimento e de ações positivas no seu contexto social vêm mostrando ser alternativas eficientes para superar as vulnerabilidades a que estão expostos (Castro, Abramovay, Rua, \& Andrade, 2001).

Experiências de protagonismo podem ser desenvolvidas por meio de programas de intervenção em prossocialidade, os quais são importantes estratégias para prevenção da violência 
e promoção da saúde mental. Tais programas objetivam amenizar os fatores de risco e contribuir para a prevenção de problemas mentais e comportamentais, dentre eles os problemas de conduta, como os comportamentos antissociais (Bandeira, Del Prette, \& Del Prette, 2006; Caprara, Steca, Zelli, \& Capanna, 2005; Escotorín \& Roche, 2010; Feitosa, Matos, Del Prette, \& Del Prette, 2009; Rocha \& Silvares, 2010; Roche, 1997; Roche \& Sol, 1998).

Os programas de intervenção em prossocialidade buscam desenvolver a prática de comportamentos prossociais que, segundo Ei- senberg (1989 citado por Carlo \& Koller, 1998) e Roche (2010), caracterizam-se por ações que visam, prioritariamente, ao benefício do outro, sem busca de recompensa externa ou material e com aceitação do receptor. Esses comportamentos foram agrupados por Roche (2010) em dez classes distintas, a saber: ajuda física; serviço físico; dar e compartilhar; ajuda verbal; consolo verbal; confirmação e valorização positiva do outro; escuta profunda; empatia; solidariedade; presença positiva e unidade. A definição das dez classes de comportamentos prossociais proposta por Roche (2010) é apresentada na Tabela 1.

\section{Tabela 1}

Definição das Dez Classes de Comportamentos Prossociais

\begin{tabular}{ll}
\hline Classes de ações prossociais & Definição \\
\hline
\end{tabular}

Ajuda física

Serviço físico

Dar e compartilhar

Ajuda verbal

Consolo verbal

Confirmação e valorização positiva do outro

Escuta profunda

Empatia

Solidariedade

Presença positiva e unidade

Os comportamentos aqui tratados são entendidos na vertente da Psicologia Positiva, a qual busca evidenciar, além dos problemas e propriedade ou uso.
Conduta não verbal que procura dar assistência a outras pessoas para cumprir um determinado objetivo, e que conta com sua aprovação.

Conduta que elimina a necessidade dos receptores da ação de intervir fisicamente no cumprimento de uma tarefa, com aprovação ou satisfação de quem recebe.

Entregar objetos, alimentos ou algo de sua posse a outras pessoas perdendo sua

Explicação ou instrução verbal; compartilhar ideias ou experiências de vida, que são úteis e desejáveis para outras pessoas ou grupos na execução de um objetivo.

Expressões verbais para reduzir a tristeza de pessoas e aumentar seu ânimo.

Expressões verbais para confirmar o valor de outras pessoas ou aumentar sua autoestima (interpretar positivamente a conduta de outros, desculpar, interceder com palavras de simpatia ou elogio).

Atitudes de atenção que expressam acolhida aos conteúdos manifestados por alguém em uma conversa.

Comportamentos que expressam compreensão dos pensamentos de alguém ou emoção de estar experimentando sentimentos parecidos aos dessa pessoa.

Comportamentos físicos ou verbais que expressam aceitação voluntária de compartilhar as consequências, especialmente penosas, da condição, status, situação ou infelicidade de outras pessoas, grupos ou países.

Presença pessoal que expressa atitude de proximidade psicológica, atenção, escuta profunda, empatia, disponibilidade para o serviço, ajuda e solidariedade para com outras pessoas e que contribui para um clima psicológico de bem estar, paz, concórdia, reciprocidade e unidade num grupo ou reunião de duas ou mais pessoas. dificuldades inerentes às etapas do ciclo vital, o desenvolvimento de habilidades e competências, como os comportamentos prossociais. Essa 
vertente busca valorizar a promoção da saúde mental, na qual, o positivo não é o oposto do negativo, ambos supõem sistemas psicológicos diferentes, no qual se preserva o reconhecimento de que as pessoas sofrem e adoecem, mas se encarregando de potenciar aquilo que funciona bem (Solano, 2010).

Levantamento realizado por Inglés, Martínez-González, Valle, García-Fernández e Ruiz-Esteban (2011) mostrou que pesquisas em Educação e Psicologia têm dado muito mais destaque para condutas agressivas e antissociais de adolescentes do que para seus aspectos de desenvolvimento. Porém, revelou que estudantes com alta prossocialidade apresentaram também maior motivação para aprender e para conseguir bons resultados acadêmicos do que os demais. $\mathrm{O}$ mesmo estudo indicou que o desenvolvimento de comportamentos prossociais contribui para a prevenção do fracasso e evasão escolar. Estudos de Caprara et al. (2005) e Koller (1997) mostraram que comportamentos prossociais podem se tornar relativamente estáveis no período da adolescência.

Em relação ao ambiente escolar, a Lei n. 9.394 (1996) que estabelece as Diretrizes e Bases da Educação Nacional Brasileira (LDB) afirma que propiciar espaços de participação, diálogo, respeito às diferenças e exercício da cidadania é primordial para a redução dos índices de violência, ainda que as escolas muitas vezes se configurem como espaços onde são vivenciados conflitos que desfavorecem o desenvolvimento positivo dos alunos (Assis, Avanci, Pesce, \& Ximenes, 2009). A relevância de se considerar a dimensão socioeconômica e cultural do entorno escolar e de configurar a escola como ambiente saudável é também destacada por Dourado e Oliveira (2009) e por Milani et al. (2006). Em estudo de Trianes, Blanca, Morena, Infante e Raya (2006), a melhora do ambiente social na escola foi associada à diminuição do risco de condutas agressivas e violentas entre os estudantes.

O Programa Mínimo de Incremento Prossocial (PMIP) criado por Roche (2010) foi aplicado em 198 estudantes de 14 a 16 anos em escolas públicas e privadas de Barcelona (Espanha). Os resultados mostraram alterações positivas significativas no comportamento prossocial individual e coletivo dos alunos, com melhora no ambiente de sala de aula, aumento na ajuda física, na conduta inclusiva e na valorização positiva do outro (Romersi, Martínez-Fernández, \& Roche, 2011).

Frente aos resultados positivos alcançados nas investigações anteriormente citadas e com a intenção de contribuir com as pesquisas acerca do desenvolvimento positivo de adolescentes, o presente estudo objetivou avaliar os comportamentos prossociais em adolescentes estudantes, com aplicação do PMIP, criado por Roche (2010).

\section{Método}

\section{Participantes}

Participaram do estudo 21 adolescentes, entre 13 e 15 anos de idade, alunos matriculados e frequentando as aulas de uma classe de $9^{\circ}$ ano do ensino fundamental, em período matutino, numa Unidade Municipal de Educação, na cidade de Santos (SP), com colaboração de uma inspetora de alunos no trabalho de campo.

O projeto foi aprovado pelo Comitê de Ética e Pesquisa da Universidade Federal de São Paulo (CEP-UNIFESP). A amostra foi selecionada por critério de conveniência e acessibilidade. A classe submetida ao estudo foi indicada pela equipe técnica da escola e as atividades do programa de intervenção foram inseridas no calendário escolar da turma.

Todos os alunos da classe participaram das atividades do programa, no entanto, foram incluídos como participantes da pesquisa apenas aqueles com autorização nos Termos de Consentimento Livre e Esclarecido (TCLE) e nos Termos de Assentimento, conforme normas éticas para pesquisas com seres humanos. Também foi definido como critério a participação em, no mínimo, $70 \%$ das atividades do programa, além de responder aos instrumentos de avaliação antes e após a intervenção. 


\section{Instrumento}

Para avaliação dos comportamentos prossociais foi utilizada a Escala de Avaliação de Prossocialidade para Adolescentes (EAP-A), em versão validada para a língua portuguesa por Oliveira-Monteiro, Aznar-Farias e Roche (2011). A EAP-A é uma adaptação do Questionário de Avaliação de Comportamento Prossocial (Roche, 2007). Trata-se de uma escala tipo likert de cinco pontos, composta por 40 questões sobre comportamentos prossociais voltados ao contexto escolar. O instrumento é autoaplicado e ainda não há estudos psicométricos de validação no Brasil.

$\mathrm{O}$ instrumento permite verificação de indicativos das dez classes de comportamentos prossociais propostas por Roche (2010) divididas em cinco categorias: Ajuda (A); Cuidado e Atenção (C); Partilha (P); Empatia (E) e Clima Positivo $(\mathrm{Cl}+)$. A categoria Ajuda é composta por oito afirmações; a categoria Cuidado e Atenção é composta por 11 afirmações; a categoria Partilha, possui oito afirmações; a categoria Empatia é composta por três afirmações e a categoria Clima positivo é composta por dez afirmações.

\section{Procedimentos de Coleta de Dados}

Os procedimentos incluíram organização conjunta com a equipe escolar para inserção da pesquisa no calendário letivo, com acordos referentes à classe escolhida, dias de encontro e definição do profissional colaborador da pesquisa, uma inspetora de alunos.

A escala foi aplicada de forma coletiva, juntamente ao Critério de Classificação Econômica Brasil, aqui denominado Critério Brasil, elaborado pela Associação Brasileira de Empresas de Pesquisa (ABEP, 2012) para caracterização da amostra. Ambos foram aplicados no início do trabalho de campo e a escala foi reaplicada após 13 semanas, período no qual foram realizadas as atividades do PMIP.

O PMIP é parte do modelo teórico-prático proposto por Roche (1995), denominado UNIPRO (uni $=$ unidade + pro $=$ prossocialidade). O modelo corresponde a uma aproximação globalizada do comportamento humano, numa abor- dagem humanista e está orientado a trabalhar sobre os critérios mínimos indispensáveis para se desenvolver competências e gerar mudanças no comportamento dos adolescentes em um breve período de tempo. O programa foi criado para aplicação em escolas secundárias com o objetivo de incrementar os comportamentos prossociais voltados para o desenvolvimento de competências sociais positivas.

O PMIP prevê a adequação das atividades a serem desenvolvidas em contextos diferentes e deve ser trabalhado a partir de três níveis, com alcance de três objetivos: sensibilização cognitiva - aumentar a sensibilidade dos participantes sobre a importância de um estilo de atuação prossocial; treinamento - conhecer e analisar as propostas para a melhora de suas relações interpessoais e; aplicação na vida real - aplicar a prossocialidade em diferentes âmbitos sociais de atuação. No presente estudo, o PMIP foi realizado em 10 sessões com os estudantes em grupo, em encontros de periodicidade semanal e duração de duas horas aula.

A Tabela 2 apresenta as ações e atividades realizadas durante todo o trabalho de campo, divididas por encontro e sessões dedicadas à intervenção.

\section{Procedimentos de Análise de Dados}

Os resultados da EAP-A foram analisados de forma descritiva, com posterior análise inferencial. Foi utilizado o teste $t$-Student pareado (Magalhães \& Lima, 2011), para comparar os resultados antes e depois da intervenção (PMIP). Foram considerados valores significativos $p \leq$ 0,05 .

A avaliação quantitativa das respostas dadas pelos adolescentes à EAP-A foi feita por frequência de respostas nas escalas tipo likert, de 1 a 5 pontos, nas quais 1 e 2 referem-se a indicativos de menor grau de prossocialidade, 4 e 5 referem-se a indicativos de maior grau de prossocialidade, e 3 refere-se a um grau médio de prossocialidade ou não indicativo de tendências para menor ou maior prossocialidade. O tratamento dos dados da EAP-A considerou a média geral dos itens investigados e o agrupamento das questões 
Tabela 2

Ações e Atividades Realizadas em Campo

\begin{tabular}{|c|c|}
\hline Encontros & Ações e atividades \\
\hline Atividades prévias & $\begin{array}{l}\text { Envio de memorando para autorização da Secretaria de Educação - SEDUC. Combinados } \\
\text { finais com a escola. }\end{array}$ \\
\hline $1^{\mathrm{o}}$ encontro & $\begin{array}{l}\text { Apresentação da proposta aos alunos. Entrega dos Termos de Consentimento Livre e } \\
\text { Esclarecido (TCLE) e Termos de Assentimento. }\end{array}$ \\
\hline $2^{\circ}$ encontro & $\begin{array}{l}\text { Recolhimento dos TCLE e Termos de Assentimento. Aplicação do instrumento de } \\
\text { avaliação EAP-A e Critério Brasil (ABEP, 2012). }\end{array}$ \\
\hline $3^{\circ}$ encontro & $\begin{array}{l}1^{\text {a }} \text { sessão de intervenção - apresentação do Programa; introdução ao tema com confecção } \\
\text { de cartazes em subgrupos. }\end{array}$ \\
\hline $4^{\mathrm{o}}$ encontro & $\begin{array}{l}2^{\mathrm{a}} \text { sessão de intervenção - uso de recurso audiovisual com apresentação do filme " } A \\
\text { corrente do bem" (Abrams, Levy, \& Leder, 2004). }\end{array}$ \\
\hline $5^{\circ}$ encontro & $\begin{array}{l}3^{a} \text { sessão de intervenção - discussão e reflexão sobre o filme com identificação de ações } \\
\text { prossociais dos personagens; levantamento de ações prossociais praticadas pelos alunos; } \\
\text { definição do conceito de prossocialidade. }\end{array}$ \\
\hline $6^{\circ}$ encontro & $\begin{array}{l}4^{\mathrm{a}} \text { sessão de intervenção - apresentação de cada aluno a partir da história de seu nome; } \\
\text { levantamento do que cada um é capaz de fazer pelos outros em sala de aula. }\end{array}$ \\
\hline $7^{\circ}$ encontro & $\begin{array}{l}5^{\text {a }} \text { sessão de intervenção - levantamento das necessidades da classe em subgrupos e do } \\
\text { compromisso de cada um para melhorar a convivência em sala de aula. }\end{array}$ \\
\hline $8^{\circ}$ encontro & $\begin{array}{l}6^{\text {a }} \text { sessão de intervenção - início do desenvolvimento de um plano de atuação pessoal na } \\
\text { escola, a partir da consciência de si mesmo. }\end{array}$ \\
\hline $9^{\circ}$ encontro & $\begin{array}{l}7^{\text {a }} \text { sessão de intervenção - levantamento do que cada um tem feito para cumprir seu plano } \\
\text { pessoal; dinâmica "Escravos de Jó" para exercício de atuação em grupo. }\end{array}$ \\
\hline $10^{\circ}$ encontro & $8^{\mathrm{a}}$ sessão de intervenção - representação teatral das ações propostas nos planos pessoais. \\
\hline $11^{\circ}$ encontro & $\begin{array}{l}9^{a} \text { sessão de intervenção - análise prossocial de conflitos a partir da história de uma } \\
\text { personagem fictícia com vivência de conflitos familiares. }\end{array}$ \\
\hline $12^{\circ}$ encontro & $\begin{array}{l}10^{\text {a }} \text { sessão de intervenção - apresentação de cartazes elaborados pela pesquisadora com } \\
\text { apanhado das atividades realizadas nas } 10 \text { sessões; avaliação das sessões por parte dos } \\
\text { alunos. }\end{array}$ \\
\hline $13^{\circ}$ encontro & Reaplicação do instrumento de avaliação EAP-A. \\
\hline $14^{\mathrm{o}}$ encontro & $\begin{array}{l}\text { Reaplicação dos instrumentos aos faltosos. Atividade de encerramento e entrega de } \\
\text { Certificados de participação. }\end{array}$ \\
\hline
\end{tabular}

do instrumento nas cinco categorias, com respeito à Ajuda (A), Cuidado e Atenção (C), Partilha (P), Empatia (E) e Clima positivo $(\mathrm{Cl}+)$.

Uma análise qualitativa dos resultados foi realizada a partir da observação e registro de manifestações comportamentais apresentadas pelo grupo de alunos considerando-se a frequência dos mesmos durante todo o trabalho de campo. Esses dados foram sistematizados em eixos temáticos referenciados pela literatura utilizada, com parâmetros de prossocialidade, de protago- nismo do adolescente e de perspectivas psicanalíticas, a saber:

Comportamentos de agitação e ansiedade - abarcando comportamentos de inquietação motora e verbal, conversas e atividades paralelas à tarefa proposta, risadas fora de contexto, dificuldades de concentração.

Dificuldades de contato com conteúdos internos - dificuldades de conexão com os próprios sentimentos e emoções, frustrações, desapontamentos, medos, expectativas, com mani- 
festação de comportamentos de recusa e esquiva de tratar certos assuntos como próprios.

Dificuldades de protagonismo - comportamentos relacionados a uma posição passiva, enquanto aluno e adolescente, sem manifestação de movimentos de autonomia mesmo com estimulação para tanto e ausência de manifestação de comportamentos proativos.

Dificuldades para trabalhar em grupo dificuldades para desenvolver tarefas grupais e ações coletivas, incluindo manifestação de estranhamento em relação às atividades propostas em grupo.

Empatia, solidariedade, sensibilidade demonstração de expressões de sensibilidade, solidariedade, abertura para aproximação e encontro, com indicativos de identificação com a vivência e o sentimento do outro, conexão emocional e possibilidade de colocar-se emocionalmente em seu lugar.

Envolvimento positivo com a tarefa proposta - comportamentos integrados afetiva e cognitivamente com as tarefas propostas, com manifestação de curiosidade, interesse, atenção e participação.

Comportamentos de agressividade - expressões verbais de acusação, comportamentos de desafio, intolerância ao diferente, desvalorização e desprezo.

Expressões de ordem depressiva - abarcando comportamentos expressivos de isolamento, ensimesmamento e retraimento durante atividades de grupo.
Manifestações de necessidade de autoafirmação - expressões indicativas e de necessidade de reconhecimento, de ser visto e valorizado, incluindo disputas/competições entre alunos e entre subgrupos, voltadas para autorreconhecimento.

Resistência às tarefas propostas - comportamentos de oposição às propostas apresentadas com demonstração de desinteresse, desconfiança, cansaço e indicativos de simulação de não entendimento, incluindo dificuldades de desenvolver as tarefas de forma lúdica.

Avanços na postura reflexiva com incremento de comportamentos prossociais - comportamentos indicativos de reflexão em relação à forma de pensar e de agir relacionados à prossocialidade, com manifestações de maior conexão com os próprios sentimentos e emoções e mudanças de postura na relação, denotando mais empatia, cuidado e acolhimento.

\section{Resultados}

A caracterização da amostra e os resultados do estudo, em seus parâmetros quantitativos e qualitativos, encontram-se apresentados nas Tabelas 3 a 5 .

Na Tabela 3, encontram-se os dados de caracterização da amostra, constituída pelo grupo de adolescentes investigados. Eles tinham de 13 a 15 anos e eram pertencentes a classes econômicas variadas, segundo o Critério Brasil (ABEP, 2012), mas com maior concentração nas classes B1 e B2.

Tabela 3

Caracterização da Amostra

\begin{tabular}{|c|c|c|c|c|c|c|}
\hline \multirow{2}{*}{ Idade } & \multirow{2}{*}{ Feminino } & \multirow{2}{*}{ Masculino } & \multirow{2}{*}{ Total } & \multicolumn{3}{|c|}{ Classe econômica } \\
\hline & & & & $\mathrm{B} 2$ & B1 & A 2 C1 C 2 D \\
\hline 13 anos & 01 & 02 & 03 & \multirow{4}{*}{$38 \%$} & \multirow{4}{*}{$28,58 \%$} & \multirow{4}{*}{$33,22 \%$} \\
\hline 14 anos & 10 & 06 & 16 & & & \\
\hline 15 anos & 01 & 01 & 02 & & & \\
\hline Total & 12 & 09 & $N=21$ & & & \\
\hline
\end{tabular}


$\mathrm{Na}$ Tabela 4 estão apresentadas as medidas descritivas e as diferenças entre as médias (+/desvio padrão) encontradas nas avaliações antes e após a aplicação do programa de intervenção, por categorias, referentes às questões respondidas pelos alunos. Os resultados mostraram que os participantes permaneceram próximos aos valores para um grau médio de prossocialidade nas categorias: Ajuda, Partilha, Cuidado e Atenção, e Empatia, antes e após a intervenção. Já na categoria Clima positivo, os resultados mostraram tendência a um melhor grau de prossocialidade, antes e após a aplicação do programa.

Tabela 4

Resultados da EAP-A Antes e Após a Aplicação do PMIP

\begin{tabular}{lcccc}
\hline \multicolumn{1}{c}{ Categorias } & $\begin{array}{c}\text { Avaliação } \\
\text { Média }(+/-D P)\end{array}$ & $\begin{array}{c}\text { Reavaliação } \\
\text { Média }(+/-D P)\end{array}$ & $\begin{array}{c}\text { Diferença média } \\
\text { Nível descritivo } \\
(\text { valor de } p)\end{array}$ \\
\hline Ajuda & $24,62(6,92)$ & $25,52(6,11)$ & $-0,94$ & 0,522 \\
Partilha & $28,38(6,20)$ & $28,71(5,27)$ & $-0,33$ & 0,805 \\
Cuidado e Atenção & $34,62(7,10)$ & $34,62(6,86)$ & 0,00 & 1,000 \\
Clima positivo & $41,67(6,29)$ & $42,00(6,86)$ & $-0,33$ & 0,819 \\
Empatia & $10,95(3,11)$ & $10,38(2,87)$ & 0,57 & 0,517 \\
\hline
\end{tabular}

Tabela 5

Principais Manifestações Comportamentais dos Estudantes Durante a Aplicação do PMIP

\begin{tabular}{|c|c|c|c|c|c|c|c|c|c|c|c|c|c|c|}
\hline 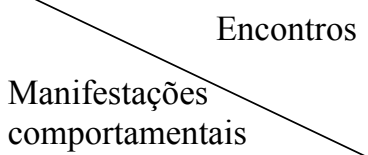 & $1^{\mathrm{o}}$ & $2^{\circ}$ & $3^{\circ}$ & $4^{\circ}$ & $5^{\circ}$ & $6^{\mathrm{o}}$ & $7^{\circ}$ & $8^{\circ}$ & $9^{\circ}$ & $10^{\circ}$ & $11^{\circ}$ & $12^{\circ}$ & $13^{\circ}$ & $14^{\circ}$ \\
\hline Agitação/ansiedade & S & $\mathrm{S}$ & S & S & $\mathrm{S}$ & & $S$ & $\mathrm{~S}$ & S & $\mathrm{S}$ & S & S & & \\
\hline $\begin{array}{l}\text { Dificuldades de contato } \\
\text { com conteúdos internos }\end{array}$ & & & & & $\mathrm{S}$ & & $\mathrm{S}$ & $\mathrm{S}$ & & $\mathrm{S}$ & & & & \\
\hline $\begin{array}{l}\text { Dificuldades de } \\
\text { protagonismo }\end{array}$ & & & S & & & & S & S & $\mathrm{S}$ & S & & $\mathrm{S}$ & & $\mathrm{S}$ \\
\hline $\begin{array}{l}\text { Dificuldades para } \\
\text { trabalhar em grupo }\end{array}$ & S & & $\mathrm{S}$ & & & & S & S & $\mathrm{S}$ & S & & & & $\mathrm{S}$ \\
\hline $\begin{array}{l}\text { Empatia, solidariedade, } \\
\text { sensibilidade }\end{array}$ & & & & $\mathrm{S}$ & S & S & & & & & S & & & \\
\hline $\begin{array}{l}\text { Envolvimento positivo } \\
\text { com a tarefa }\end{array}$ & S & S & S & S & S & S & S & S & & S & S & S & S & S \\
\hline Agressividade verbal & & & S & & & & $S$ & & $S$ & & $S$ & & & S \\
\hline $\begin{array}{l}\text { Manifestações de ordem } \\
\text { depressiva }\end{array}$ & & & & & & & & S & & & & $\mathrm{S}$ & & $\mathrm{S}$ \\
\hline $\begin{array}{l}\text { Necessidade de } \\
\text { autoafirmação }\end{array}$ & & & S & & & S & S & & & & & $\mathrm{S}$ & & \\
\hline $\begin{array}{l}\text { Resistência às tarefas } \\
\text { propostas }\end{array}$ & & S & $\mathrm{S}$ & & S & & & $S$ & S & S & & & & S \\
\hline $\begin{array}{l}\text { Avanços na } \\
\text { postura reflexiva } \\
\text { e comportamentos } \\
\text { prossociais }\end{array}$ & & & & & $\mathrm{S}$ & & & & & & S & S & S & S \\
\hline
\end{tabular}


A Tabela 5 apresenta dados qualitativos relativos às principais manifestações comportamentais do grupo de alunos, por eixos temáticos, durante as 14 sessões realizadas. Os dados indicam manifestações de envolvimento positivo com as tarefas propostas, em quase todas as sessões, que conviveram com manifestações frequentes de agitação e ansiedade, resistências às tarefas propostas e também dificuldades para trabalhar em grupo e para exercer protagonismo. Dados de avanços na postura reflexiva e comportamentos prossociais estivem mais presentes nos últimos encontros do grupo.

\section{Discussão}

O objetivo deste estudo foi avaliar comportamentos prossociais num grupo de adolescentes estudantes, antes e após uma intervenção com uso de um programa mínimo de incremento de prossocialidade.

Embora não tenha sido detectada diferença significativa em nenhuma das categorias da escala de avaliação de prossocialidade que foi aplicada, nos dois distintos momentos da avaliação, os dados qualitativos indicaram tendências de incremento nos comportamentos prossociais após aplicação do programa de intervenção e experimentação de um novo papel social, por parte dos estudantes, dentro do ambiente escolar.

Manifestações comportamentais indicativas de envolvimento positivo com as tarefas propostas estiveram presentes em praticamente todos os encontros, denotando interesse e curiosidade acerca da experimentação de novos papéis sociais, conforme referido por Erikson (1990) e Levy (2001) na literatura de adolescência apresentada. Por outro lado, durante aplicação da dinâmica "Escravos de Jó" (na sétima sessão de intervenção), os participantes mostraram maior dificuldade para realização da tarefa grupal com manifestações de críticas e de agressividade verbal diante dos colegas que eram menos ágeis e que tinham menor coordenação e ritmo, habilidades exigidas no jogo. Conforme Levy (2001), tais reações ocorreram possivelmente também geradas pela experimentação de um novo papel social na relação com seus pares, indicando vi- vências grupais que tanto apoiam atitudes como expressam intensamente crítica, característica comum nesse período do desenvolvimento.

$\mathrm{Na} 4^{\mathrm{a}}$ sessão do PMIP, na qual aconteceram as maiores trocas empáticas a partir de histórias pessoais compartilhadas e acolhidas pela pesquisadora e pelo grupo, os participantes mostraram interação grupal positiva, denotando exercício da prática de convivência e aprendizado com seus pares, conforme apontaram Milani et al. (2006) e também Trianes et al. (1997), caracterizando a vivência grupal na experimentação de novos papéis sociais como parte da formação da identidade. Nesse encontro, os participantes manifestaram maior sensibilidade, empatia e solidariedade com os demais, indicando condições favoráveis para efetivação de comportamentos prossociais, conforme apontaram estudos de Romersi et al. (2011).

Em aproximadamente metade dos encontros, mesmo estimulados pelas atividades propostas, foi observada ausência de comportamentos proativos nos estudantes, indicando dificuldade do grupo para o exercício do protagonismo. Esse dado foi observado com maior intensidade nas sessões de intervenção especialmente dedicadas à desenvoltura de planos pessoais e de ações grupais de exercício da prossocialidade.

Houve indicativos de manifestação de conflitos entre o desejo de participar de forma atuante e colaboradora e uma posição passiva diante da proposta. Em alguns momentos, os estudantes expressaram posição contrária e desafiadora ao proposto, sugerindo contestar o mundo adulto e suas regras, de acordo com o apontado por Levy (2001), quanto ao período da adolescência. Porém, as dificuldades para exercer o protagonismo e para trabalhar em grupo, presentes em metade dos encontros realizados, podem ser refletidas também no tocante ao contexto da instituição escolar na qual aconteceu o trabalho de pesquisa.

De acordo com a LDB (Lei n. 9.394, 1996), as unidades de ensino do Brasil ainda apresentam limitações para a prática de uma educação mais participativa e protagonista de seus alunos, dado também abordado nos estudos de Assis et al. (2009), nos quais as autoras apontaram que nem sempre a escola favorece o desenvolvimen- 
to positivo dos seus alunos, contrariando o que é indicado pelo MS (2010) para o incremento de ações protagonistas nos adolescentes como um fator que pode favorecer a sustentabilidade das estratégias de saúde e o desenvolvimento comunitário.

As atividades do programa de intervenção que tinham o intuito de provocar postura mais ativa e desenvolvimento da autonomia e do protagonismo dos alunos pareciam novidades dentro da cultura institucional, causando aparente estranhamento no grupo. Em contrapartida, apesar das dificuldades acima descritas, a instituição escolar mostrou-se com grande potencial para incremento de medidas de proteção dos recursos pessoais, de sentimentos de pertencimento e interação dos adolescentes com diferentes sistemas ambientais, como também foi apontado nos estudos de Koller (1997), Roche $(2007,2010)$ e Roche e Sol (1998).

As manifestações de ordem depressiva e os indicativos de necessidade de reconhecimento e autoafirmação observados pareceram indicar estados de preocupação voltados a si mesmos e busca por serem vistos e valorizados em suas características pessoais na vivência do enfrentamento de estados de solidão decorrente da aquisição de autonomia, com presença de crises e confusão de papéis, características da construção da identidade na adolescência, conforme apontaram Erikson (1990) e Levy (2001). Já no momento da autoavaliação, após a aplicação do programa de intervenção, o grupo de alunos mostrou-se mais confiante diante da pesquisadora e dos seus pares, fazendo menor uso de mecanismos de defesa diante da atividade proposta e denotando maior contato com conteúdos internos, além de estabelecimento de vínculo afetivo com a pesquisadora e com o grupo de iguais. Esse resultado indicou possibilidade de ampliação da auto-percepção e maior potencial para reconhecer aspectos menos favoráveis acerca de si mesmos, conforme apontou Roche (2010) quanto ao potencial do PMIP. Milani et al. (2006), Roche (1997, 2007, 2010) e Romersi et al. (2011) afirmaram que o autoconhecimento favorece o desenvolvimento de habilidades sociais, aumentando também o grau de prossocialidade.
A dinâmica de alteração nas manifestações dos estados emocionais apresentada pelo grupo de alunos mostrou-se compatível com o período de constituição de identidade e própria da adolescência (Erikson, 1990; Levy, 2001; Milani et al., 2006; Shoen-Ferreira et al., 2010; Trianes et al., 1997). Nos encontros finais da intervenção houve indicativos de avanços na postura reflexiva para incremento de comportamentos prossociais, como apontado por Romersi et al. (2011) quanto ao uso do PMIP. Tais apontamentos indicam que, apesar dos resultados quantitativos não apresentarem alterações significativas após a aplicação do programa, a análise qualitativa dos resultados apontou favorecimento de possibilidades reais de experimentação de um novo lugar dentro da sala de aula por parte dos adolescentes, com possíveis desdobramentos para o espaço escolar, a família e a comunidade.

Quanto a não presença de alterações nos resultados quantitativos, frise-se que o grupo de estudantes já apresentava graus médios de prossocialidade no início do estudo, dificultando a detecção de diferenças significativas entre os dois momentos. Por outro lado, essa característica da amostra favoreceu a análise qualitativa. Os avanços na postura reflexiva dos alunos ao final dos encontros de intervenção, denotando maiores possibilidades de incremento dos comportamentos prossociais, coadunam com a literatura apresentada a respeito do PMIP, quando indica que este pode ser uma alternativa para abrandar consequências negativas decorrentes de possíveis vulnerabilidades inerentes à própria etapa da adolescência e ao contexto social em que os adolescentes estão inseridos.

\section{Considerações Finais}

Conforme apontado anteriormente, o estudo aqui tratado buscou contribuir com a ampliação de investigações acerca do desenvolvimento positivo de adolescentes, especificamente no campo dos comportamentos prossocias. Tais comportamentos podem se constituir como recursos protetivos nas interações dos adolescentes com seus contextos de desenvolvimento e abarcam desde sistemas mais proximais até aqueles perti- 
nentes a interações sócio-tecnológicas, como no caso das interações em redes.

Embora sem alterações significativas nos resultados quantitativos pertinentes a comportamentos prossociais, a análise qualitativa dos comportamentos apresentados durante as sessões do programa de incremento à prossocialidade (abarcando atividades grupais, posturas de convivência nos encontros de intervenção, as trocas empáticas e os avanços na postura reflexiva acerca dos comportamentos prossociais) chama a atenção para características peculiares da adolescência num enfoque positivo e favorece a abertura de um leque para outras tantas possibilidades de investigação.

Considera-se que a principal limitação do estudo foi o número reduzido da amostra. Também a presença muito discreta de pesquisas com a temática da prossocialidade e, mais especificamente, a ausência de outros estudos com utilização do mesmo programa de intervenção na população brasileira, foram elementos restritivos para avanço e amplitude das discussões no rol de trabalhos dessa natureza. Entretanto, um ponto positivo a ser considerado no trabalho foi a possibilidade de investigação dentro de uma unidade pública de ensino, com superação de obstáculos relativos a pouca flexibilização das grades curriculares, com necessidades de ajustes do calendário escolar aos prazos acadêmicos.

Para ampliação e aprofundamento de estudos sobre a temática dos comportamentos prossociais em adolescentes sugere-se a continuidade de investigações que abarquem amostras maiores, populações com queixas de problemas de comportamento e graus baixos de prossocialidade, estudos de comparação entre populações com queixas de comportamentos e sem queixas submetidas ao mesmo programa, avaliações de prossocialidade feita pares (outros estudantes), além de trabalho no campo com mais de um pesquisador. Sugere-se também avaliação do próprio programa de intervenção utilizado em seus aspectos objetivos e de ordem qualitativa, o qual se revelou com potencial para favorecer o desenvolvimento da prossocialidade em adolescentes.

\section{Referências}

Abrams, S. R. P., Levy, R. L. (Produtores), \& Leder, M. (Diretora). (2004). A corrente do bem [Filme]. Burbank, CA: Warner Bros Entertainment.

Assis, S. G., Avanci, J. Q., Pesce, R. P., \& Ximenes, L. F. (2009). Situação de crianças e adolescentes brasileiros em relação à saúde mental e à violência. Ciência e Saúde Coletiva, 14(2), 349-361. doi:10.1590/S1413-81232009000200002

Associação Brasileira de Empresas de Pesquisa. (2012). Critério de Classificação Econômica Brasil. São Paulo, SP: Autor.

Bandeira, M., Del Prette, Z. A. P., \& Del Prette, A. (Eds.). (2006). Estudos sobre habilidades sociais e relacionamento interpessoal. São Paulo, SP: Casa do Psicólogo.

Bronfenbrenner, U. (2001). A Ecologia do Desenvolvimento Humano: Experimentos naturais e planejados (2. ed.). Porto Alegre, RS: Artmed.

Caprara, G. V., Steca, P., Zelli, A., \& Capanna, C. (2005). A new scale for mesasuring adults prosocialness. European Journal of Psychological Assesment, 12(2), 77-89. doi:10.1027/10155759.21.2.77

Carlo, G., \& Koller, S. H. (1998). Desenvolvimento moral pró-social em crianças e adolescentes: Conceitos, metodologias e pesquisas no Brasil. Psicologia: Teoria e Pesquisa, 14(2), 161-172.

Castro, M. G., Abramovay, M., Rua, M. G., \& Andrade, E. R. (2001). Cultivando vida, desarmando violências: Experiências em educação, cultura, lazer, esporte e cidadania com jovens em situação de pobreza (Relatório técnico). Brasília, DF: Banco Interamericano de Desenvolvimento.

Dourado, L. F., \& Oliveira, J. F. (2009). A qualidade da educação: Perspectivas e desafios. Caderno CEDES, 29(78), 201-215. doi:10.1590/S010132622009000200004

Erikson, E. H. (1990). El ciclo vital completado. Buenos Aires, Argentina: Paidós.

Escotorín, P., \& Roche, O. R. (2010). Cómo y por qué prosocializar la atención sanitária: reflexiones, desafios y propuestas (Conclusiones del Proyecto Europeo CHANGE/2010). Barcelona, España: Fundação Universitária Martíl'Humá.

Feitosa, F. B., Matos, M. G., Del Prette, Z. A. P., \& Del Prette, A. (2009). Desempenho acadêmico e interpessoal em adolescentes portugueses. Psi- 
cologia em Estudo, 14(2), 259-266. doi:10.1590/ S1413-73722009000200006

Inglés, C. J., Martínez-González, A. E., Valle, A., García-Fernández, J. M., \& Ruiz-Esteban, C. (2011). Conduta prosocial e motivação acadêmica em estudantes espanhóis de educação secundária obrigatória. Universitas Psychologica, $10(2), 451-465$.

Koller, S. H. (1997). Educação para a pró-sociabilidade: Uma lição de cidadania? Paidéia (Ribeirão Preto), 12-13, 39-50. doi:10.1590/S0103$-863 X 1997000100004$

Lei n. 9.394, de 20 de dezembro de 1996. (1996, 23 dez.). Estabelece as Diretrizes e Bases da Educação Nacional. Diário Oficial da União, seção 1.

Levy, R. (2001). O adolescente. In C. L. Eizirik, F. Kapczinski, \& A. M. S. Bassols (Eds.), O ciclo da vida humana: Uma perspective psicodinâmica (pp. 127-140). Porto Alegre, RS: Artmed.

Magalhães, M. N., \& Lima, A. C. P. (2011). Noções de probabilidade e estatística (7. ed.). São Paulo, SP: Editora da Universidade de São Paulo.

Milani, F. M., Jesus, R. C. D. P., \& Bastos, A. C. S. (2006). Cultura de paz e ambiências saudáveis em contextos educacionais: A emergência do adolescente protagonista. Educação (Porto Alegre), 2(59), 369-386.

Ministério da Saúde. (2010). Diretrizes nacionais para a atenção integral à saúde de adolescentes e jovens na promoção, proteção e recuperação da saúde. Série A. Normas e Manuais Técnicos/2010. Brasília, DF: Secretaria de Atenção em Saúde, Departamento de Ações Programáticas Estratégicas.

Oliveira-Monteiro, N. R., Aznar-Farias, M. A., \& Roche, R. O. (2011). Escala de Avaliação de Prossocialidade para Adolescentes (EAP-A). Manuscrito não publicado.

Rocha, M. M., \& Silvares, E. F. M. (2010). Competências de jovens brasileiros: Fator de proteção para problemas comportamentais. In Actas do VII Simpósio Nacional de Investigação em Psicologia (pp. 1162-1171). Braga, Portugal: Universidade do Minho.

Roche, R. (1995). Psicologia y educacion para la prosocialidad: ciencia y técnica. Barcelona, España: Universidad Autónoma de Barcelona.
Roche, O. R. (1997). Educacion prosocial de las emociones, actitudes y valores en la adolescencia. Barcelona, España: Universitat Autónoma de Barcelona.

Roche, O. R. (2007). Desarrollo de la inteligencia emocional y social desde los valores y actitudes prosociales em la escuela. Buenos Aires, Argentina: Ciudad Nueva.

Roche, O. R. (Ed.). (2010). Prosocialidad nuevos desafios: métodos y pautas para la optimización creativa del entorno. Buenos Aires, Argentina: Ciudad Nueva.

Roche, O. R., \& Sol, N. (1998). Educación prosocial de las emociones, valores y actitudes positivas para adolescentes en entornos familiares y escolares. Barcelona, España: Blume.

Romersi, S., Martínez-Fernández, J. R., \& Roche, R. (2011). Efectos del Programa Mínimo de Incremento Prosocial en una muestra de estudiantes de educación secundaria. Anales de Psicología da Universidade de Murcia, 27(1), 135-146.

Shoen-Ferreira, T. H., Aznar-Farias, M., \& Silvares, E. F. M. (2010). Adolescência através dos séculos. Psicologia: Teoria e Pesquisa, 26(2), 227234. doi:10.1590/S0102-37722010000200004

Solano, A. C. (Ed.). (2010). Fundamentos de psicologia positiva. Buenos Aires, Argentina: Paidós.

Trianes, M. V., Blanca, M. J., Morena, L., Infante, L., \& Raya, S. (2006). Um cuestionário para evaluar el clima social del centro escolar. Psicothema, $18(2), 272-277$.

Trianes, M. V. T., Muñoz, A. M., \& Jimenez, M. (1997). Competencia social: su educación y tratamiento. Madrid, España: Pirámide.

Zacarés, J. J., \& Serra, E. (1998). La madurez personal: perspectivas desde la psicología. Madrid, España: Pirámides.

Recebido: 09/01/2014

$1^{a}$ revisão: 02/05/2014

$2^{a}$ revisão: $29 / 08 / 2014$

$3^{a}$ revisão: $22 / 09 / 2014$

$4^{a}$ revisão: 03/10/2014

Aceite final: 08/10/2014 